\title{
History of EPR Studies from the H.M. Swartz Laboratories: Part 1-Free Radicals and Paramagnetic Metals in Biological Systems and Associated EPR Instrumental Developments
}

\author{
Harold M. Swartz ${ }^{1}$ (D)
}

Published online: 5 January 2022

(c) The Author(s), under exclusive licence to Springer-Verlag GmbH Austria, part of Springer Nature 2021

\section{Personal Overview of Harold M. Swartz (HMS)}

\subsection{HMS' Initial Training and Paths Leading to Studies in EPR}

My introduction to EPR came in my third year as an active duty medical officer in the US Army. The specific focus of my work there was based on the unique capabilities of EPR to specifically detect and characterize free radicals and other species with unpaired electrons. The specific problem was to determine if the radioprotective drugs, based on aminothiols, worked by the mechanism of moderating radiation damage through modifying free radical production and/subsequent reactions of the products from the initial reactions with free radicals. While the literature on the reactions of aminothiols and other sulfhydryl (SH)-containing compounds usually started with statements of the well-known reactivity of SH groups with free radicals, a review of the literature traced down this "well-known fact" to only a suggestion that had been made in one publication. However, after a series of citations were made to this observation in different articles, those articles were subsequently cited as proof of this 'well-established fact', and the original hypothetical basis of the 'fact' was lost. This non-experimental evolution from a hypothetical to accepted fact was an eye-opening experience that permanently imprinted on me the need to understand the evidentiary basis of common assumptions in research (and clinical medicine as well). However, this is getting somewhat ahead of my story.

Fortunately, my medical school years at the University of Illinois School of Medicine at Chicago and my internship at Michael Reese Hospital as well as the first 2 years in the Army provided me with some very important learning opportunities in the practical applications of medicine and public health that helped inform my subsequent use of and passion for applying EPR to solve biological/medical problems.

Harold M. Swartz

Harold.Swartz@Dartmouth.edu

1 Department of Radiology, Geisel School of Medicine, Dartmouth College, Hanover, NH, USA 
During my first year in the Army, I was assigned to a remote military post in Dugway, Utah, deep into the desert some 90 miles from Salt Lake City and in a different world. The base was set up for field testing of chemical, biological, and nuclear $(\mathrm{CBN})$ threats. My main responsibility was as a member of a team of five physicians who provided all the medical care for the military and civilian population who lived on the base. However, I also was assigned to go to the field during testing of the dispersal characteristics of chemical agents, to provide emergency medical intervention if the wind changed and the testing crew became exposed. Situated in a tiny trailer nearby to where the testing team was deployed, my training consisted of instructions to check for the responsiveness to light of the pupils of anyone potentially exposed to nerve gas and to administer atropine if they were not responsive. Although I never needed to use this "training", waiting to apply this training during $\mathrm{CBN}$ exercises was not an experience that I would wish to repeat!

Most pertinent, I also participated in several courses taught to military officers on the military aspects of CBN warfare. I had already been interested in the biological effects of ionizing radiation and had read a fair amount about it, so I greatly enjoyed and learned from this experience. Consequently, I applied and was accepted to a special program developed to provide an intense education in the biological effects of ionizing radiation to medical officers. So after 1 year at Dugway, at my request I was sent to the "nuclear medicine" training program at the University of North Carolina at Chapel Hill (UNC) where the course work consisted principally of intense training in radiation biology and radiation physics. As a part of my program, I also earned a Master of Science in Public Health, which necessitated taking several valuable courses in public health and has provided further bonuses to my academic training and career. This educational year was topped off by a 2-month tour to various federal sites carrying out research and testing of weapons using radiation and the biological effects of the radiation.

For my third year in the Army, I was assigned to the Walter Reed Army Institute for Research (WRAIR) in Washington, DC, to work in the program trying to develop drugs that would protect the military and civilians from the effects of ionizing radiation. As a part of this larger endeavor at WRAIR, the EPR laboratory had been established with the principal rationale to use EPR to study the mechanisms of aminothiols to provide an evidentiary basis for the development of improved radioprotective drugs. However, before I arrived, no one had been assigned to have as their principal responsibility to carry out these studies. (I was soon to become its director and principal scientist.)

When I arrived at WRAIR, I was told that I would be rotated through the various labs and then a mutual decision would be made by me and the director of the program (Dave Jacobus) as to where I would have my primary assignment. My initial rotation was in the EPR laboratory (officially known as the Magnetic Resonance Lab, since it had a Varian A 60 NMR as well as a Varian V 3500 EPR). Although I had never even heard of EPR before I arrived, it quickly became apparent to me that EPR was potentially a very productive technique, and although learning it would be a challenge, I felt I was up to the task, especially because of the education in physics I had just completed at the UNC program. Therefore, after an initial few weeks in 
the EPR lab (and before I could rotate through any of the other laboratories), I asked to be and was permanently assigned to the EPR-based project.

Initially, the other personnel assigned to the EPR project consisted of one technician, a Private, who by virtue of his diligent drinking had worked himself down from Captain (attaining this rank via a field promotion) to the lowest level of Private. He did know how to run the instruments, however, and did a very good job in teaching me the essentials of their operation. One day he offered me some advice I have not followed. Slurring a bit, he confessed, "You know, Doc, I find that the instruments have less noise if I have a few drinks before operating them". Eventually, I lost his assistance, after he was discharged when he passed out drunk in the bushes outside the Commandant's house. Fortunately, in the meantime, several additional personnel had been assigned to work with me including two well-trained Ph.D.'s in EPR and NMR (both civilians), plus a series of talented technicians (all draftees, often with a good technical background).

\subsection{Factors That Shaped HMS' Scientific Approach}

Several factors in addition to my training and initial experiences have shaped my personal approaches to scientific studies. One emphasis in my scientific studies has emerged particularly from my diverse background in medicine, physics, and chemistry. I have always sought to relate rigorous physical-chemical data to valid biological endpoints, particularly those in functioning biological systems, i.e., within in vivo settings.

Another emphasis in my scientific approach is to seek ways to overcome the complications from trying to collect data within the dynamic environment of living organisms. I have tried to accomplish this by observing the changes caused by biological processes in the organisms and then leveraging the resulting physical-chemical data to obtain additional information about the biological processes.

Finally, because of my medical background and continued clinical interests, my approach from the beginning has been to design and conduct research with the explicit goal of developing methods and uncovering implications that can lead to practical and important clinical applications. This medical orientation also proved to be a valuable asset in achieving credibility and an advantage in seeking external funding. Adding a Ph.D. in biophysics (from the Dept of Biochemistry at Georgetown University during my years at WRAIR) also helped.

\subsection{Self-Teaching in EPR-Related Research and Critical Thinking}

As part of my self-training, I endeavored to master the literature on free radicals in biological systems (See our 1998 review $^{233}$ for a more complete appraisal of the following.). I quickly encountered the early emphasis on hypotheses that free radicals had important roles in causing pathophysiology. The basis for this was a vague concept that, because free radicals caused some unusual reactions, they might play an important role in causing diseases whose origin was not well understood, especially cancer, and they might also be the basis of aging. While some considerations had 
a rational basis, many hypotheses were very dubious. This path led to my becoming familiar with some pretty outlandish theories and questionable interpretations of results. Consequently, I voiced my concerns about them at scientific meetings and other forums and undertook to carry out more rigorous studies to demonstrate what was actually happening with free radicals in biological systems.

This experience led to my broader skepticism whenever reviewing studies with surprising findings, especially those using NMR as well as EPR. Consequently, some of my publications and organization of meetings have focused on resolving controversies in science.

My approach for free radicals has been by attempting to establish more plausible mechanisms for the observed phenomena and to try to determine more precisely the actual roles for free radicals. Consequently, we have published a number of articles disproving some hypotheses that had been put forward on whether free radicals cause cancer and other diseases ${ }^{22-24,29,30,42,47,53-56,61,64,65,73,74,86}$ and aging ${ }^{215}$. My studies in cancer, both when trying to disprove some theories and establish what roles free radicals could play in biological systems, were in some ways gratifying, and the scientific discussions at international meetings were sometimes quite colorful.

Arguably, however, looking at the number of my publications devoted to putting down incorrect hypotheses and interpretations of data, this may not the best way to advance translation of scientific findings into real world clinical applications. On the other hand, in retrospect, some of my articles that were especially valuable for the field focused on resolving uncertainties, paradoxes or outright errors in the literature; see especially two of my publications in Science ${ }^{3,42}$. Most importantly, I have come to appreciate that one of the most important qualities in one's publications (and in mentoring) is to retain a critical attitude, not only toward the results and explanations of others, but especially toward one's own results and interpretations.

\section{Review of HMS' Laboratory Publications in Free Radicals}

\subsection{General Historical Approach to This 'Review' of My Work in Free Radicals}

Very soon after the discovery of EPR in 1944 by the Soviet scientist, Evgenii Konstantinovic Zavoisky, a number of experimentalists tried to use this capability to test existing theories of roles of free radicals in pathophysiology and physiology, and this in turn generated additional theories. However, the application of EPR to biology was very challenging because the existing EPR techniques, which were based on the availability of microwave techniques developed from the radar programs of WWII, lost sensitivity in the presence of liquid water due to non-resonant absorption by the electrical component of the electromagnetic microwaves used in radar, i.e., $9 \mathrm{GHz}$ (often termed X-Band).

Consequently, because liquid water is intrinsic to almost all life, steps to minimize its influence not only had the potential to change the biology, but also could introduce unanticipated artifacts. The procedures employed were removal of liquid water (by lyophilization or direct drying) or immobilizing it by freezing (frozen 
water does not cause non-resonant losses). A less perturbing approach was to leave the water in place but use very thin samples which could be oriented within the resonant cavity to a position where the electric field was a minimum while the magnet field was maintained. However, this strategy introduced problems of maintaining the biological environment (e.g., level of oxygen and glucose, elimination of metabolic products, alterations of $\mathrm{pH}$ ).

As summarized below, we were able to make some contributions to such experimental approaches, especially to explain that many of the results were problematic, but also to add some positive information. The eventual and very productive and satisfying approach was to participate in the development of methods to make EPR measurements in vivo, primarily by developing and utilizing lower frequency EPR (about $1 \mathrm{GHz}$ ), which enabled the studies to be done in intact, functioning animals and then in human subjects. These developments required the contributions of a range of collaborators with skills in EPR instrumentation, with many of the key people coming from a very productive collaboration with the highly capable group of scientists in Krakow, Poland, Tad Sarna, Wojiech Froncisz, Tad Walczak, Piotr Lesniewski, and Karol (W. K.) Subczynski and several key contributors from the former Union of Soviet Socialist Republics (USSR) including Alex Smirnov, Oleg Grinberg, and Ildar Salikhov.

\subsection{Overview of My Work in Free Radicals}

To tell the story about my group's efforts in free radicals, it is useful to categorize the work before discussing it in more detail. ${ }^{1}$ Our investigations involving free radicals included:

- Use of potential modifiers of free radicals to reduce the impact of ionizing radiation.

- EPR signals occurring naturally in biological systems. (These studies eventually involved several different areas, and therefore, will be considered separately.)

o Free radicals occurring in the process of metabolism and/or related to specific pathophysiology (especially cancer)

o The role of ascorbic acid on the EPR signals seen in cells after lyophilization or direct drying

o Free radicals in melanin (a stable free radical)

o Paramagnetic metal ions (initially these spectra were mistakenly attributed to free radicals and then were studied deliberately)

- Use of introduced free radicals, usually nitroxides or their derivatives, to study physiologically and pathophysiologically important processes.

\footnotetext{
1 Details are included below, with references to the relevant publications involving our group. Note that references to work from my labs use a universal numbering system across all three histories in this series. They are noted in superscript and can be found in 'Overview of Issue 2, Acknowledgements and References' in this festschrift ${ }^{576}$.
} 

o Nitroxides
o Spin traps

- Development of instrumentation for following free radicals in vivo.

\subsection{Historical Perspectives of Our Publications Involving Free Radicals in Biological Systems}

\subsubsection{Using Potential Modifiers of Free Radicals to Reduce the Impact of lonizing Radiation}

We started by trying to study how aminothiols modify free radicals by measuring biologically valid endpoints (such as survival of irradiated cells) in parallel with following the modifications of radiation-induced free radicals. This rapidly became quite complex when we began by trying to duplicate the key changes seen by Smaller and Avery [1] on the effect of radioprotectors on the EPR signal seen in irradiated yeast. The first tasks in replicating their work were, therefore, to develop an experimental system to grow the yeast the way that they did, to obtain the EPR spectra the same way, and in parallel to measure the impact of radiation on the viability of the yeast.

The experimental complications became apparent when we realized that they had obtained more resolved EPR spectra using fully deuterated glucose as the energy source. However, this product was not available commercially, having been made specially for them at Argonne National Laboratory. Since deuteration was key to resolving the spectra, we eventually succeeded using a work-around. We bought fully deuterated water, and when the yeast was grown in it, they made deuterated glucose for us.

The next problem was that when we froze the yeast, survival without radiation was less than $1 \%$. With such poor survival rates under the best condition, it seemed undesirable to try to measure the impact of radiation under the various conditions we had planned.

Therefore, while completing the complex steps to be able to finish the long delayed experiments with yeast to demonstrate that we were able to reproduce the previously published results of Smaller and Avery, we started down a parallel path of experiments using a bacterial strain that had 85-100\% survival with fast freezing. We were as a consequence able to publish a series of papers in which we examined the inter-relationships among chemical structures of the aminothiols, their impact on radiation-induced free radicals, and most importantly, we related these to the biological effect of interest ${ }^{5,8-10,16}$. These papers showed that there was a direct correlation between the impact of the aminothiols on the radiation-induced free radicals and the amount of protection against radiation. We found a gradual decrease in the efficacy of protection as the distance between the amino and thiol groups was increased. It should be noted, however, that while these correlations supported the suggested mechanism for the observations, they did not rigorously prove it. 
We never carried out further studies on the radioprotectants. Nonetheless, throughout my career, I have drawn on the knowledge I gained about radiation protectants and the biological mechanisms of radiation damage. This has been especially important in our studies on biodosimetry ${ }^{571}$ and in our studies on oxygen ${ }^{572}$. The latter started with the observation in the experiments with the isolated cells and radioprotectants that, when the cells were frozen in oxygen-containing media, not only was there a greater impact of radiation damage but there was also an impact on survival from the freezing itself. This observation became the basis of my thesis research for my Ph.D. and is described more completely in the accompanying paper on the history of studies of the oxygen effect ${ }^{572}$.

While I was working on overcoming the problems on achieving my main task, I carried out several more readily implemented experiments with EPR, looking at the interactions of ionizing radiation with tissues. For reasons noted above, chronologically, these papers preceded publishing our findings on the aminothiols. The first such 'side study' focused on a different problem that reflected my fascination with radiation physics and took advantage of the central role that the nuclear reactor played in my department at WRAIR (Biophysics). The goal was to establish a better way to measure the penetration of neutrons into tissues, which was limited largely by the lack of a model for tissues that fully reflected the composition of real tissues. I reasoned that a quick solution might be to bypass the models and use real tissue itself. To overcome the problems with the changes that would occur at room temperature, I carried out the studies on deeply frozen tissue, i.e., in a dog frozen close to liquid nitrogen temperatures.

This approach was feasible because the initial absorption of the neutrons should be independent of temperature or state of the water. I followed the flux of thermal neutrons through tissues using the relatively high threshold of sodium atoms for absorption of thermal neutrons. This could be done simply by measuring the induced radioactivity in sodium from absorption of the neutrons. I also used EPR to relate the production of radicals by the neutrons by keeping the tissue deeply frozen and measuring the yield of the thermally immobilized free radicals ${ }^{1}$.

This work led logically to a second "side study', i.e., carrying out a "control" experiment with the aim of confirming the instability of the radiation-induced free radicals. The rats were first irradiated in vivo at room temperature and then the sacrificed animals were frozen so that the tissues could be measured at low temperatures. To our surprise, we found that there were some very long-lived free radicals ${ }^{2,4}$. This finding led to perhaps our most cited paper $^{6}$ where we hypothesized that these very long-lived free radicals in tissues such as teeth and bone could be utilized for afterthe-fact biodosimetry of subjects whose exposure was unknown. This "incidental" work subsequently became the basis for what turned out to be a very major part of my research career, as detailed in a separate history on my laboratories' work in biodosimetry ${ }^{571}$.

\subsubsection{EPR Signals Occurring Naturally in Biological Systems}

The first subtopic of our publications in this area involves the roles of free radicals that occur in the processes of metabolism and/or related to specific pathophysiology. 
Following many years of conjecture about the role of free radicals in physiology (normal processes) and pathophysiology (disease), EPR studies that had the potential to directly test these hypotheses began with the work of Barry Commoner [2]. Commoner was especially interested in the potential roles of free radicals in metabolism. Soon after Commoner's ground-laying work, a number of publications and meetings continued to add evidence on the same subject, while expanding into models to investigate roles of free radicals in enzymatic mechanisms.

Of note in this area, an extensive set of studies using EPR in biological systems were carried out in the USSR during this period. Unfortunately, for scientific discourse, they had minimal impact outside of the USSR. In particular, their use of lyophilized samples to overcome the water in biological samples (which decreased their applicability to biological systems), combined with the low sensitivity of most EPR instruments in the USSR and their lack of details about the experiments that typified most publications from the USSR led to their neglect by most scientists in the rest of the world.

As I tried to become familiar with the early literature on EPR studies in normal tissues, I became aware of what appeared to be very different results from different laboratories [3-8]. We were able to sort out the basis for the apparent contradictions, which turned out to be their use of different experimental approaches. In particular, they used conditions that resulted in very different levels of effective microwave in the samples. The results of this investigation were considered significant enough to be published in Science $^{3}$. Subsequently, throughout my research career with EPR, I have been very cognizant of how microwave power saturation could not only be a potential source of confusion but more importantly, when used deliberately, could be a powerful spectroscopic tool.

In this same area, with the help of two brilliant EPR scientists, Jim Bolton and Don Borg, we put together a comprehensive review of a large body of data on EPR studies of cells and tissues as part of a book on the Biological Applications of Electron Spin Resonance ${ }^{19-21}$. Over almost 50 intervening years and still ongoing, I have been gratified to hear very positive comments about the continuing value of this now very old book (1972!) Our group carried out some additional studies of naturally occurring free radicals in tissues, especially liver ${ }^{13,46}$, and using multifrequency spectroscopy, in the cervix and uterus ${ }^{83}$.

Similarly, another review of the technique of EPR with an emphasis on biological applications continues to be acknowledged as informative. This was done with a very bright college student (my elder daughter, Sharon Swartz, who is now a very well recognized Professor of Evolutionary Biology at Brown University). Since she had had no training in magnetic resonance, the explanations in this review were able to be deliberately written so as to make sense to interested people outside of the field ${ }^{66}$.

We did carry out some direct studies of metabolic free radicals, with an emphasis on using these to link to redox status. This approach was based on a fairly substantial set of studies by others of enzymes in model systems, which indicated that the level of free radicals reflected the level of redox related metabolism. We assumed that the $g=2$ signal, which readily saturated, indicated the level of biochemical free radical intermediates of enzymatic reactions. 
We used this approach to study the impact of nitrous oxide on metabolism in the kidney and liver ${ }^{13}$. We found that nitrous oxide depressed the free radical level in the liver but not in the kidney. The mechanism for this became apparent as a part of controlled experiments we conducted. Because nitrous oxide depresses eating, the resulting fasting increased the free radical level in the liver but not in the kidney, consistent with the role of the liver to control the availability of energy sources. Similarly, in the heart we used the levels of the metabolic free radicals as a tool to measure the functioning of the myocardium when it was stressed ${ }^{182}$.

The second subtopic of publications from my laboratories involved the naturally occurring roles of free radicals in the pathophysiology of cancer. My group carried out several studies investigating a speculative but fairly widespread theory that free radicals have a prominent role in carcinogenesis and cancer. In retrospect, while this concept was superficially attractive, a careful examination of the data and the underlying theory led to the conclusion that there was no substantial basis for these theories. In the process of sorting this out, we carried out a number of careful studies in which we made EPR measurements of tissues during and after the development of cancer. I believe that our most important contribution to this field was to publish both the data and conceptual overviews indicating the problems in the free radical theory of cancer ${ }^{22-24,29,30,42,47,53-56,61,64,65,73,74,86}$.

Our final conclusion about the role of free radicals in cancer is summarized concisely in the abstract of my chapter in a book entitled Magnetic Resonance in Cancer $^{86}$ :

Electron Spin Resonance (ESR or EPR) is a technique that specifically and sensitively detects chemical species with unpaired electrons, including free radicals and certain valence states of transition elements. ESR studies of cancer initially were undertaken to test theories postulating intrinsic roles of free radicals in cancer. Such theories have never been substantiated and in view of the current state of knowledge of cancer, it seems unlikely that they shall be. Similarly, there appear to be no general relationships between paramagnetic metals and cancer, in spite of some early reports that there might be such a relationship.

In the process of carrying out and reporting these studies I had a number of very entertaining opportunities to interact with some pretty far-out people who were convinced that free radicals were the basis of cancer (and perhaps everything else bad that that happened to people). Closely related to this were our contributions to try to debunk the concept of free radicals as the basis of aging in an article entitled "Free radicals in aging: Theories, facts, and artifacts," which was not very well-received by many of the participants at a conference whose purpose was to promote the theory of free radical aging ${ }^{215}$.

The third subtopic of our publications studying free radicals in naturally occurring biological systems involved the role of ascorbic acid on the EPR signals seen in cells after lyophilization or direct drying.

As noted before, lyophilization was used especially in the USSR. While this resulted sometimes in robust EPR signals, it was soon recognized that the observed signals were likely to be artifactual. A few Soviet scientists, especially Enno Ruuge 
and Lev Blumenfeld, carefully and competently addressed this problem, sorting out the conditions that were required to avoid many of the artifacts.

In a series of six papers, in collaboration with Nick Dodd from Manchester, England, we were able to show that most of the EPR signals seen with lyophilized or dried biological samples could be attributed to ascorbic acid radicals $33,53,55,63,64,73$.

The fifth subtopic involved free radicals in melanin. Melanin is the only widespread biological molecule that has a naturally occurring free radical. My entry into this important and fascinating area came about accidently, in the process of my organization in 1974 of the first Gordon Conference that covered the Biological Importance of Free Radicals in Biology and Medicine. The organizing focus was my extremely naive idea that it would be possible to resolve the many controversies about the role of free radicals in cancer at a Gordon Conference, with its combination of formal and informal discourse and 24/7 week-long emersion into food, relaxing and science. In addition, this Conference was to help determine whether NMR could provide a specific indication of the presence of cancer.

My naivety rested on my assumption that, if only we would get together all the various scientific parties to these controversies in a scientific but informal environment such as the Gordon Conferences, everyone would rapidly become familiar with each other's approaches and evidence and could thereby form a scientific consensus that would emerge after a week spent in such an environment. While no such consensus emerged, the outcome of this effort was a very interesting start towards a series of events that had a large impact on my career and that of many others.

The full story is too long and complex to cover here, but one result was my introduction to the amazing EPR group at the Jagiellonian University in Krakow, Poland which led to many important developments in EPR. Most pertinent to the present history is my introduction to a bright young scientist from Krakow, Tadeusz Sarna. We have subsequently collaborated extensively, including a joint paper in this special edition $^{570}$. Since that paper reviews much of the work in melanin with which I have been involved $35,37,38,50,57,58,68,72,75,76,105,114,159,178-180,185,186,210,217,219,221,249,337$, I will discuss here only some aspects that it does not cover.

One aspect we exploited was to use the EPR signal of melanin to show some practical applications in identifying pigments that develop in pathophysiology $34,49,50,89,98,179,192$. We were able to resolve a controversy about whether the pigment involved in the Dubin-Johnson syndrome is melanin (it is not $)^{34,49,89}$. Another was to determine the nature of the pigment associated with side effects from the antibiotic, minocycline ${ }^{98,192}$. More generally, we showed how EPR could help in the identification of the origins of unknown pigments ${ }^{179}$, including undifferentiated tumors ${ }^{50}$. Another set of important studies looked at the impact of melanin on the response of cells to irradiation, in which we showed that it was the nature of the cells and not the melanin that determined cellular response ${ }^{75}$.

The sixth subtopic is paramagnetic metal ions. The initial interest in paramagnetic metal ions grew from mistakenly attributing their EPR spectra to the presence of free radicals. Once this mistaken observation was corrected, studies then deliberately focused on understanding their nature and implications. Our initial paper (in Science) demonstrated that EPR spectra of paramagnetic metal ions could be observed in cells and tissues, if the spectroscopic conditions were appropriate ${ }^{3}$. This 
could be observed most readily when measurements were made at low temperatures because the relaxation times of the metal ions were sufficiently prolonged that they were observable. Later, we were also able to show that some paramagnetic ions, especially manganese $2+$, had sufficiently long relaxation times that they could be observed at room temperature ${ }^{20}$.

Over the next several years, we conducted a series of studies in which we observed EPR signals in biological systems that turned out to be due to paramagnetic metal ions, some of which turned out to have practical applications. One particular application arose from our interest in EPR signals measured in organs destined for transplant, especially the liver.

The initial basis for these studies was to determine if the free radical signals associated with the metabolic processes could be an indicator of the viability of the tissues. While we did find an EPR signal that appeared to correlate with the viability of liver for use in transplant, we discovered that the EPR signal was probably due to a molybdenum ion in an enzyme system whose modification was empirically correlated with the viability of the liver ${ }^{17,18}$.

We later generalized this correlation to a number of other metal ion EPR signals $^{25}$. Our argument was that the basis for these correlations, as cells become damaged, is that they lose their ability to maintain redox stability, and therefore, the metals in enzymes change their oxidation state.

Somewhat analogously, while investigating theories that free radicals were involved in cancer (discussed further below), we observed a number of systems where their EPR spectra changed in the presence of cancer. Many of these EPR spectral changes turned out to be due to changes in paramagnetic metal ions ${ }^{22-24,29,30}$.

We also found some interesting other sources of paramagnetic spectra in biological samples that turned out to be introduced by contaminants. Two examples were contamination (chromium complexes) that occurred from histological fixatives ${ }^{36}$ and contamination (copper-dithiocarbamate complex) from rubber gloves ${ }^{38}$.

When we developed in vivo EPR we were able to follow the metabolism of metal ions whose oxidation state, and hence their EPR spectra, changed in vivo 209,251,287. This included the ability to determine the conversion of chromium to an oxidation state associated with carcinogenesis.

\section{Introduced Free Radicals, Usually Nitroxides or Derivatives, Used to Study Physiological or Pathophysiological Processes}

Since most biological systems do not have naturally occurring EPR-detectable free radicals (meaning free radicals that are sufficiently long-lived during physiological processes and that are detectable at in vivo temperatures), stable free radicals need to be introduced to allow probing with the powerful capabilities of EPR. This need to introduce stable free radicals raises both opportunities and challenges. While the very productive, classical uses of spin labels in quasi-biological systems such as model membranes already had considered the potential physical and chemical perturbations of the systems that were being studied, such considerations were often not done thoroughly when spin labels were used in more fully functional biological 
systems such as cells or even organelles. The opportunities when extending the technique to use functioning biological systems include the amazing amount of information that one can obtain from the EPR spectra of the introduced probes, especially the influences from their immediate environment. For example, in addition to information about their physical structure, EPR can detect oxygen levels, metabolism, $\mathrm{pH}$, etc.

Most of our work has been done with nitroxides as the introduced free radical. These extraordinarily important and versatile molecular structures have had an immense impact on biological applications of EPR. Nitroxides have unpaired electrons associated with sterically hindered NO groups attached to carbon atoms. The EPR spectra reflect the interactions of the unpaired electrons with the environment. Potentially, their spectra can provide an enormous amount of information about the nature of the environment that is difficult if not impossible to obtain by other techniques.

We have focused on efforts with nitroxides for applications for EPR studies of functioning biological systems and as contrast agents for use in MRI. This has been accomplished by exploiting the chemical versatility of the nitroxides and their metabolism in actively functioning biological systems. We also used a different type of spin label, nitric oxide, to study the structure of hemoglobins ${ }^{28}$. Initially we carried out a number of spin label studies in living cells. We developed the methodology to obtain data in living cells under physiological conditions, thereby obtaining very useful functional information difficult to obtain by other means $59,60,70,79,80,118,237$. The challenges were complex and include the stability of the probes in the challenging environment of functional biological systems, the potential perturbations introduced by the probes, and obtaining adequate signal intensity. We also carried out studies in model membranes in collaboration with Kocherginsky ${ }^{226,237}$.

Our work in this area initially focused on how to get valid data in the face of the metabolism of the nitroxides. We later recognized that, if we instead focused on understanding the metabolism, we could exploit the interactions to further our understanding about how the interactions, both physical and biological, affected their stability and location in vivo. This information could then be used to get more information from EPR spectroscopy. It also led to their use as contrast agents for MRI as discussed below.

There have been two lines in our research investigating the biological interactions of nitroxides. The first examined metabolic interactions, their potential toxicity, and how to use interactions to measure function and pathophysiology in cells, organs, and in vivo. We, therefore, have devoted considerable time and effort to understanding their interactions with the biological systems, both to obtain maximum information and to minimize unrecognized perturbations.

We had the good fortune to be able to draw on the expertise and productivity of the outstanding scientists who conceived the nitroxide structures, made them in immense varieties, and developed methods to physical and chemical methods characterize their behavior and model their spectra. Therefore, we undertook to extensively measure the impact of cellular functions/nitroxide interactions as a primary component of many of our studies, i.e., we used the interactions of nitroxides to study a wide range of physiological and pathophysiological 
processes $92,95,97,99,115,116,118,120-124,128,132,135,137,140,143,144,148,152,155,156,162,164,191,196,198$, 199,235,252,254-263,267,279,302,307,309,364,379. We summarized our published and some unpublished data in 1995 in a comprehensive book Nitroxide Spin Labels, Reactions in Biology and Chemistry ${ }^{226}$. Now more than 25 years old, this book remains a principal source of information on the use of nitroxides and of spin traps in functional biological systems.

As noted previously, the potential "problem" of the spin label being metabolized can actually be a potent source of additional information, especially about the processes that influence the metabolism of nitroxides. Because oxidation/reduction within viable biological systems is both important and difficult to measure in vivo by other techniques, we have particularly focused on exploiting their metabolism as a means to probe oxidation/reduction in vivo. We have already published an overview and summary of our research in this field up to $2005^{367}$.

To assay redox status with nitroxides, we examined changes in the redox state by measuring the rates of reduction of nitroxides to non-paramagnetic hydroxylamines by the disappearance of the EPR signal. We examined changes in the status of the oxidation state by measuring the rates of oxidation of hydroxylamines to paramagnetic nitroxides, observing the generation of an EPR signal of the nitroxide. We also have used SH-sensitive nitroxides as an assay for the availability of SH groups, which are a major factor in the redox status of cells. We have continued to expand the use of this concept, as summarized elsewhere ${ }^{168,379,431,528}$. As detailed elsewhere in the history of oximetry ${ }^{572}$, we also can follow oxygen levels, which of course are a very crucial part of redox reactions ${ }^{167}$. Since all these assays can be done in vivo with EPR and with no other technique, I believe that this is a very important and still underutilized capability of in vivo EPR.

Using nitroxides in studies of pharmacokinetics and other aspects of pharmaceuticals represents another type of major application. These have been done in collaborations with Bernard Gallez and Karsten Mäder who spent 1-2 years in our lab before returning to their academic careers in pharmacy in Belgium and Germany, respectively. Together we demonstrated that EPR could be a powerful tool in this field $141,235,277,279$.

The second line of our investigations using nitroxides to measure physiological and pathophysiological function in vivo was in their use as contrast agents for MRI. I consider this to be a very important major application that has the potential to have a major impact because of the widespread availability and clinical use of MRI.

Heretofore, the principal use of MRI had been to obtain information on anatomy. The idea behind using nitroxides as a contrast agent for MRI is fairly simple but powerful: output from measurements should reflect not just the anatomy, but more interestingly, the physiological or pathophysiological function. Although this idea to add functional information had been recognized by others prior to our work, I believe that our explicit conceptualization and practical demonstration of its utility was a major accomplishment. Specifically, we introduced the concept of combining the use of contrast agents with parameters of biological functioning using nitroxides. When others had used nitroxides as contrast agents for MRI, they had focused on their chemical versatility. 
When a contrast agent was needed for anatomical imaging in NMR, paramagnetic metal ions were usually selected instead of nitroxides. This was because of the mechanism by which they interact with the protons of water. While nitroxides interact via outer shell electrons, metal ions impact nuclear relaxation more directly and were considered to be the better choice.

We carried out our studies to exploit these mechanisms of how nitroxides interact with the protons of water in collaboration with Seymour Koenig, the leading expert in mechanisms of MRI relaxation for contrast ${ }^{82,88,94,130}$. We were able to add a new dimension to MRI contrast using nitroxides as probes for important biological functions (like metabolism). We also used the physical properties of nitroxides, such as their lipophilicity, to be able to localize them selectively. This information could in turn be used to edit NMR spectra to be able to measure lactic acid by eliminating the overlapping signals from lipids ${ }^{160}$.

We note that other approaches in MRI, based on acquisition parameters rather than contrast agents, have increasingly focused on additional ways to measure function. These other approaches are complementary to, not competitive with, our approach to use nitroxides as contrast agents.

The principal ideas of our approach are summarized in several key papers in which we demonstrated that one could use the metabolic and physical properties of nitroxides to obtain contrast in MRI that reflects function ${ }^{99,108,169,367}$. In parallel, again in collaboration with Seymour Koenig, we carried out rigorous studies of the nature of their impact on nuclear relaxation ${ }^{68,72,102}$. These studies included the impact of oxygen on the relaxation of nuclei, an approach which, after an interval of many years, was picked up by the MRI community to become an active field of investigation to measure oxygen in vivo using MRI.

The second subtopic regarding our investigations into introduced free radicals to study physiological or pathophysiological processes involved spin traps. Our studies using spin traps involved three overlapping applications: classical spin trapping (including characterization of spin traps and their adducts), use of spin traps in vivo, and novel uses of spin traps $51,106,109,110,155,227,230,231,265,290,293,299,304,310,314,316,317,364,368,389,434$.

The initial classical spin trapping study enabled us to resolve the question of what species were produced when carbon tetrachloride was metabolized by the liver. We also carried out several studies to characterize the interactions of spin traps with functional biological systems, including metabolism of the initial products of spin trapping and determining the relationships between structure of spin traps and their stability in functioning cells. Perhaps most uniquely, we developed both the methods and the instrumentation to enable measurements of spin traps in vivo ${ }^{231,310,314}$.

An interesting development that we have not pursued further yet, but which already has been productive, has been to use EPR imaging to measure cell viability, using nitroxides with quenching by paramagnetic metals ${ }^{146}$. Viable cells exclude the paramagnetic metal ions, so intracellular nitroxides can be imaged if the cells are viable and cannot be imaged if the cells are damaged. 


\section{Development of Instrumentation for Following Free Radicals In Vivo}

During our studies aimed at solving important biological questions, we (i.e., usually the many talented engineers with whom I have had the good fortune to be associated) often had to advance the state of the art of EPR spectroscopy. Innovative advancements were especially necessary because, to achieve our experimental goals, we often needed to make the studies under biologically pertinent conditions. That is, EPR needed to be able to make measurements in living cells or in intact organisms and at their usual temperature and in their usual micro-environments including with appropriate levels of nutrients, oxygen and $\mathrm{pH}$. We also developed techniques for EPR imaging 99,105,115, working in collaboration with Paul Lauterbur (who was co-awarded the 2003 Nobel Prize in Medicine or Physiology for his discoveries concerning magnetic resonance imaging).

Most important, both in terms of its impact on the field and the direction of much of our research, was our development of techniques to make measurements in vivo in animals (especially humans!) These instrumental developments were led by two outstanding engineers who were originally from Krakow, Tadeus (Ted) Walczak and Piotr Lesniewski. Our publications in this field included both techniques for use in functional biological systems and the development of the instrumentation needed to make the measurements $21,39,45,52,66,69,77,151,174$, $181,187,189,200,206,212-214,216,224,234,238-242,251,268,273-275,286,315,332,354,370,381,390-395,484,495,505$ $, 521,531,550$

Some of these instrumental developments occurred in the context of measuring radiation dose in vivo ${ }^{571}$, but these results were applicable for medically oriented in vivo EPR as well. The medical applications have particularly focused on measurements of oxygen in vivo. These aspects are discussed in more detail in an accompanying paper on the history of our developments to measure oxygen in vivo ${ }^{572}$.

We made a conscious choice to carry out our developments for in vivo measurements at L-Band ( $1200 \mathrm{MHz}$ frequency and 400 gauss magnetic field) and with an emphasis on spectroscopy rather than imaging. The choice of L-Band as our frequency was made as a compromise between sensitivity (which favors higher frequencies) and depth at which in vivo measurements could be made (which favors lower frequency). We chose to focus on spectroscopy rather than imaging to obtain the desired information as quickly as possible because we wanted to follow physiological and pathophysiological processes, which are very time-dependent.

The initial instrumental development focused on needs for applications in small rodents, i.e., mice or small rats. Consequently, we were able to adapt existing electromagnets and just lower the magnetic fields by modifying the current to the main coils. Our major developments were in the development of a very sensitive and reliable microwave bridge and in the development of suitable resonators. Both required extensive developments, but fortunately we had Ted Walczak initially and then also Piotr Lesniewski to carry these out. Eventually, they developed a L-Band microwave bridge whose performance has yet to be equaled. The bridge has been improved 
even further in collaboration with Wojciech Froncisz and his colleagues, both within our laboratory and in the lab in Krakow ${ }^{567}$.

The resonators were even more challenging because, for in vivo applications, our usual approach is to place the resonator on the surface of the subject. Over many years, Ted and Piotr and others such as Hiroshi Hirata worked on coaxing out the maximum signal-to-noise from the surface resonator. See articles included in this special issue for an in-depth discussion of the challenges and solutions for all types of resonator development ${ }^{555}$ and for the most recent developments of a surface resonator designed specifically for clinical $u^{561}$ as well as several overall improvements made to the instrumentation, including miniaturization ${ }^{524,567}$.

New instrumental challenges arose as we moved towards clinical applications. Early on we realized that the most extensive and impactful medical applications would focus on the ability of EPR oximetry to make direct and repeated measurements of oxygen. Measurements of free radicals, especially nitroxides and spin traps, would also be very valuable clinically, but there will be a challenge to obtain approval for the use of these materials if injected into human subjects because of the time and expense required for getting FDA approval for their use. The accompanying summary on the development of oximetry ${ }^{572}$ describes the parallel developments of the EPR oximetric materials, which were equally important for robust clinical applications.

To make measurements in larger animals or human subjects, a much wider gap is needed to place the subject in the magnetic field. (For nail biodosimetry, we looked at the possibility of using single surface magnets but were not able to obtain a design that extended the magnetic field sufficiently far from the surface of the magnet.) Such a wide gap would require a very large electromagnet with very high power requirements. We decided that it would be much more practical to use a permanent magnet which would be simpler to build and would be easier to place and operate, avoiding the need for a very large power supply and cooling for the magnet. This did, however, raise the problem of potential thermal drifting. We concluded that we could overcome this through automated tuning. We also recognized that the large mass of a magnet with dimensions large enough to accommodate the whole body of a human would provide a pretty robust heat sink which would minimize drifting. In practice, we have indeed found that thermal drift has not been a significant problem.

We spent a lot of time and effort in improving the ergonomic aspects of the clinical system, to meet the challenge of measuring sick people rapidly and comfortably while maintaining precise positioning of the resonator. (Many important aspects of this effort are summarized in this issue ${ }^{567}$ and other publications ${ }^{525,526,537}$. We also have developed plans for special purpose clinical magnets, especially for peripheral vascular disease; for instance, a magnet for the latter disease would need to encompass only a leg and foot. 


\section{Summary and Conclusions}

The study of free radicals by EPR has evolved over the years. While the initial motivation was based on understanding the role that free radicals might have in physiology and disease, the current emphasis has both narrowed and expanded. Examples of the field's narrowing include the reduction of the prominence of free radical theories of cancer and the recognition that many key reactions formerly labeled as "free radical damage" are actually due to non-radical oxidative processes. Examples of its expansion include the widespread use of EPR in oximetry and biodosimetry, and the field's large and continuing growth is illustrated by the success of the Society for Redox Biology and Medicine (the successor to the Society for Free Radical Biology and Medicine).

The primary naturally occurring free radicals under active study by other groups are principally those potentially associated with oxidative damage. Studies of melanin free radicals also have continued to be quite productive. The use of stable free radicals, especially nitroxides, spin traps and radicals that can be used to measure oxygen, continue to be very important.

The field has to some extent bifurcated between studies in animal models and in human subjects. In animal models, one can use the full range of stable free radicals as well as study metabolic free radicals. These types of studies provide invaluable information about disease and physiology, with a considerable emphasis on oxidative stress but using stable free radicals predominantly. In human subjects, the applications that dominate are oximetry and radiation dosimetry; indeed, these area have become so important that separate histories on their development are a part of this special issue ${ }^{571,572}$. As illustrated in all the studies in this review and in the two special issues, the powerful technique of using EPR to study biological systems has made major contributions to scientific understanding as well as practical applications in clinical medicine, and these will only increase in number and importance in the future.

Acknowledgements These studies and instrumental developments are the product of many people's efforts as well as funding from many sources throughout the past 50 or so years. While I have named several special contributors in context in these histories, please see the many coauthors of my papers for a fuller picture of the key players. I am also pleased that so many of the young scientists with whom I have worked in my laboratories have gone on to have leading roles in their institutions and create their own legacies, many of whom have contributed articles for these two special issues. Last, I would like to acknowledge the always helpful criticism and editorial contributions to these histories of my most important partner and colleague (and wife): Ann Barry Flood.

Editors' Note Since there is considerable overlap in references in the three histories of EPR studies from H.M. Swartz laboratories, references with Swartz as an author use a common numbering system from HMS' curriculum vita. These numbers are noted by superscript numbers in this text, and the full references are published only in the accompanying editorial, Overview of Issue 2, Acknowledgements and References [9]. References included below with this history are those that do not include Swartz as a coauthor. 


\section{References}

Note: References in brackets included with this history are those that do not include Swartz as a coauthor. See the accompanying editorial 576 for the Swartz reference listings that are in superscript in the text.

1. B. Smaller, E.C. Avery, Nature 183, 539 (1959)

2. B. Commoner, J. Townsend, G.E. Pake, Nature 174, 689 (1954)

3. B. Commoner, J.L. Ternberg, Proc. Natl. Acad. Sci. USA 47, 1374 (1961)

4. G.A. Kerkut, M.L. Edwards, K. Leech, K.A. Munday, Experetia 17, 497 (1961)

5. D.W. Nebert, H.S. Mason, Cancer Res. 23, 823 (1963)

6. J. Mallard, M. Kent, Nature 204, 1192 (1964)

7. J. Mallard, M. Kent, Nature 210, 588 (1966)

8. J. Mallard, M. Kent, Phys. Med. Biol. 14, 373 (1969)

9. A.B. Flood, S.G. Swarts, M.C. Krishna, B. Gallez, Appl. Mag. Res. (2022). https://doi.org/10.1007/ s00723-021-01459-3

Publisher's Note Springer Nature remains neutral with regard to jurisdictional claims in published maps and institutional affiliations. 